\title{
Effects of Surface Charges on the Seawater Desalination Using Functionalized Graphene
}

\author{
Yue Chan', Yong Ren ${ }^{2}$ \\ ${ }^{1}$ School of Mathematical Sciences, The University of Nottingham, Ningbo, China \\ ${ }^{2}$ Department of Mechanical, Materials and Manufacturing Engineering, The University of Nottingham, \\ Ninbgo, China \\ Email: yue.chan@nottingham.edu.cn, yong.ren@nottingham.edu.cn
}

Received 11 January 2016; accepted 6 April 2016; published 13 April 2016

\section{Abstract}

In this paper, we investigate the selection rule for desalinating seawater using functionalized graphene sheet as a semi-permissible membrane. Both the applied mathematical modeling and MD simulations will be used to determine the acceptance conditions for water molecule or sodium ion permeating into the functionalized graphene. Both the Lennard-Jones potential and Coulomb forces are considered by taking into accounts the major molecular and ionic interactions between molecules, ions and functionalized graphene sheet. The continuous approximation will then be used to coarse grain most significant molecular and ionic interactions so that the multi-body problems could be simplified into several two-body problems and the 3D motions are reduced into degenerated 1D motion. Our mathematical model and simulations show that the negatively charged graphene always accepts sodium ions and water; however the permeability of water molecules and sodium ions becomes very sensitive to the presence of positive charges on the graphene.

\section{Keywords}

Mathematical Modeling, Continuous Approximation, MD Simulations, Salt Water, Desalination, Functionalized Graphene Sheet

\section{Introduction}

Graphene oxide comprises graphene sheets which are decorated by certain functional groups such as hydrophilic oxygen [1]. Recently, numerous computational simulations have been performed on the ultra-filtration using nanotubes [2]-[9], zeolite [10] and graphene [11] [12]. All these theoretical investigations reveal a rapid and effective ultra-filtration using nanomaterials in comparison to conventional membrane. Reverse osmosis has found to possess the merit of low energy consumption and operation costs in comparison to other desalination methods such as multi-stage flash, multi-effect distillation and mechanical vapor compression. Although most theoretical investigations reveal a superior filtration performance of nanomaterials, none of these nanomaterials have successfully been proved for the desalination. Recently, Tanugi \& Grossman [12] have nominated porous graphene as a mean for the seawater desalination due to its superior mechanical strength and the negligible thickness, and 
they claim that such graphene possesses the highest water permeability over all existing commercial RO and zeolite [12]. Besides, several experiments have paved a way to producing and transferring singly layered graphene sheets [13], which make this 2D-nano structure more applicable for large scale desalination.

However, the computational approach adopted by Tanugi \& Crossman [12] and other computational simulations [2]-[10] have severe temporal and spatial constraints that are infeasible to tackle this ultra-filtration problem in longer run and larger scale. Here, we adopt the continuous approximation introduced by Cox et al. [14] [15], where the pairwise interactions between ions, molecules and functional groups are coarse grained so that the time-consuming calculations could be fasten by performing certain line integrals, and single and double surface integrals. The present authors have successfully applied such approach on solving seawater desalination problems using functionalized nanotube and graphene [11] [16]-[19].

In this paper, we investigate the possibilities and limitations of using functionalized graphene sheet for the saltwater desalination. Carbon atoms are assumed to be smeared across the surface of the graphene sheet, and the water and sodium ion are smeared across an envisaged surface of radius 1 and $1.16 \AA$, respectively, so that the van der Waals interactions between any two ions/molecules could be approximated by performing surface integrals. Chloride ion is neglected because its ionic size is larger than the pore size of the graphene. In addition, the functional groups are assumed to be evenly distributed around the pore boundary so that the electrostatic interactions between the intruders and the functional groups are evaluated in terms of line integrals. Least but not last the interactions between molecules/ions and solute are tabulated from MD simulations results. Such approach has been shown to speed up computational times dramatically and yield accurate results. The resulting force fields could then be incorporated into the MD algorithm to determine the time evolution of the physical system numerically.

\section{Theory and MD Simulations}

In this section, we introduce the theoretical and numerical basis for the rest of the paper. For simplicity, we model the van der Waals interactions between a water molecule or a ion and the functionalized graphene by the usual 6 - 12 Lennard-Jones potential [22], and the electrostatic interactions arising from the functional group by electrostatic potential $E$, which are given, respectively by

$$
V\left(\rho_{i j}\right)=4 \epsilon\left[-\left(\frac{\sigma}{\rho_{i j}}\right)^{6}+\left(\frac{\sigma}{\rho_{i j}}\right)^{12}\right]=-\frac{A}{\rho_{i j}^{6}}+\frac{B}{\rho_{i j}^{12}}, \quad E\left(\rho_{i j}\right)=\frac{Q_{i} Q_{j}}{\varepsilon \rho_{i j}^{2}},
$$

where $\rho_{i j}, \varepsilon, \sigma, Q_{i}, \varepsilon, A$ and $B$ denote the atomic distance between two typical atoms, the Lennard-Jones potential well depth, the Lennard-Jones distance between two atoms, total charge for $i$ atom /functional group, the permittivity, the attractive constant and repulsive constant, respectively. The numerical values for several constants are summarized in [17] [19]. We assume that the carbon atoms are smeared across the graphene surface, and the oxygen atom and the sodium ion are smeared across the envisaged sphere of radius 1 and $1.16 \AA$, respectively so that the van der Waals forces between the oxygen or the sodium ion and the graphene sheet could be approximated by the double surface integrals, while the van der Waals forces between the hydrogen atom (point particle) and the graphene are determined by the single surface integral, which are given respectively by

$$
F_{2}^{v d W}(R)=\eta_{1} \eta_{2} \int\left\{-\left.\frac{d V(\rho)}{d \rho}\right|_{\perp}\right\} d S_{1} d S_{2}, \quad F_{2}^{v d W}(R)=\eta_{1} \int\left\{-\left.\frac{d V(\rho)}{d \rho}\right|_{\perp}\right\} d S_{1},
$$

where $\eta_{1}, \eta_{2},-\mathrm{d} V / \mathrm{d} \rho \mid \perp, \mathrm{d} S_{1}$ and $\mathrm{d} S_{2}$ denote the number density of carbon atoms on the graphene, the number density of the envisaged sphere, the axial force generated by the Lennard-Jones potential given in Equation (1), the surface element of the graphene and the surface element of the envisaged sphere, respectively. On the other hand, the functional group is assumed to be smeared on the pore vicinity so that the electrostatic forces between water or sodium ion and the functional group is given by

$$
F_{i}^{e}=\int_{\ell} \frac{q_{i} \sigma}{\varepsilon \rho_{\perp}^{2}} d \ell
$$

where $q_{i}, \sigma$ and $\mathrm{d} l$ denote the charge of the intruder, the charge density on the pore vicinity and the length element of the pore, respectively. We comment that all the above integrals reduce the multi-body interactions into 
several two-body problems and the introduction of the axial forces reduces the 3D problem into degenerated 1D problem. According to the prior works done by the present authors [16]-[19], certain parts of these integrals, i.e. Equations (2) and (3) could be solved analytically facilitating rapid computational times. Since the orientation of water molecules is found to be crucial in this problem [20], we initially fix the configuration of the two hydrogens separated by an angle $2 \phi$ as shown in Figure 1 and different orientations of the water molecule could be generated by two independents angles $\theta$ in $[0, \pi]$ and $\psi$ in $[0,2 \pi]$ so that the kinematics between the first hydrogen, i.e. upper hydrogen in Figure 1 and an arbitrary point on the graphene is written as

$$
\begin{aligned}
& \rho^{2}=a^{2}+\ell^{2}+R^{2}+2 a R(\cos \phi \cos \theta-\sin \phi \sin \theta \cos \psi) \\
& -2 a \ell \sin \theta \sin \psi \cos \Phi-2 a \ell(\sin \phi \cos \theta+\cos \phi \sin \theta \cos \psi) \sin \Phi,
\end{aligned}
$$

where $a, l, R, \phi$ and $\Phi$ denote the radius of the water molecule, the distance between the pore center and an arbitrary point on the graphene, the distance between the center of the water molecule and the pore center, half of the angle between two hydrogens and the polar angle on the graphene, respectively (see Figure 1 for details). The distance between the oxygen or the sodium ion and the graphene sheet or functional group can be deduced in a similar fashion. Such atomic distances could then be incorporated into Equations (2) and (3) to determine $F_{1}{ }^{\mathrm{vdW}}, F_{2}{ }^{\mathrm{vdW}}$ and $F_{\mathrm{i}}{ }^{\mathrm{e}}$. In particular, since the forces between the hydrogen atoms and the functionalized graphene are generated by different orientations, the ensemble forces acting on the two hydrogens could be deduced using Boltzmann's statistics

$$
F_{H_{i}}=\frac{\sum_{j}\left\{F_{H_{i}}(R)_{j} \exp \left(-\beta V_{H_{i}}(R)_{j}\right)\right\}}{\sum_{j} \exp \left(-\beta V_{H_{i}}(R)_{j}\right)},
$$

where $j, F_{H i}(R)_{j}, \beta$ and $V_{H i}(R)_{j}$ denote the different orientations, forces of $H_{i}$ in $j$ configuration, the reciprocal of the usual Boltzmann's constant times the temperature and the potential energy between $H_{i}$ and the functionalized graphene, respectively. The total molecular forces between the water molecule and the functionalized graphene is therefore written as

$$
F^{\text {tot }}=F_{O}^{v d W}+F_{H_{1}}^{v d W}+F_{H_{2}}^{v d W}+F_{O}^{e}+F_{H_{1}}^{e}+F_{H_{2}}^{e}-\nabla \Phi+F_{\text {hydra }}+F_{\text {app }}=F_{W}-\nabla \Phi+F_{e x t},
$$

where $F_{i}^{v d W}$ and $F_{i}^{e}$ denote the van der Waals forces between the $i$ atom, where $I=O, H_{1}$ and $H_{2}$ and the graphene and the electrostatic forces between $i$ atom and the functional group on the pore, respectively. In addition, $\Phi, F_{\text {hydra }}, F_{\text {app }}, F_{\mathrm{W}}$ denote the electric field generated by the charges on the entire graphene, the hydraulic forces in the solute, the applied force and the molecular forces comprising van der Waals and electrostatic forces between the water molecule and the graphene, respectively. Here, the external forces $F_{e x t}$ is also coarse grained by the sum of both the hydraulic and applied forces. It is worthy to note that the hydraulic forces are interpolated from experimental data or simulation results. The total force between the sodium ion and the functionalized graphene sheet can be deduced in a similar way, i.e. $F^{\text {tot }}=F_{I}-\Delta \Phi+F_{e x t}$, where $F_{I}$ denotes the ionic forces between the ion and the functionalized graphene. Given these force fields $F^{\text {tot }}$, the time evolution of the system can be numerically determined using Verlet algorithm [21]

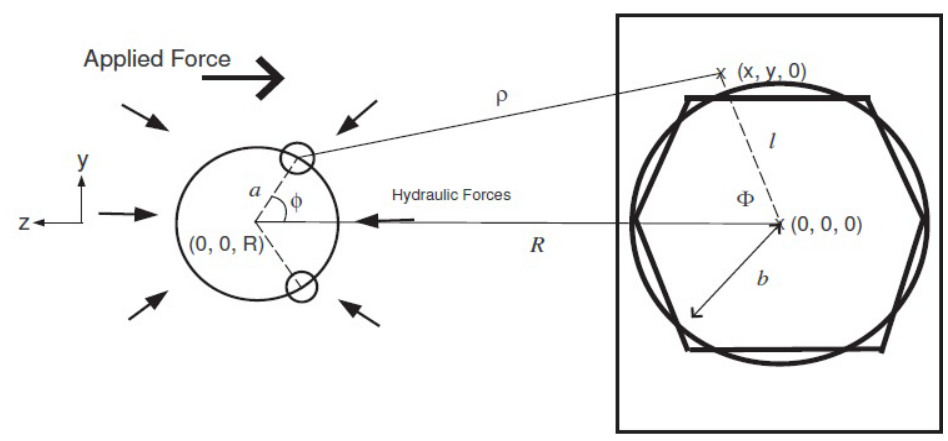

Figure 1. Kinematics of a water molecule intruding axially into a pore on the graphene, where $\rho$ denotes the atomic distance between a hydrogen atom and an arbitrary point on the graphene sheet, and the functional group is assumed to be smeared on the ring of the pore entry. Both the applied and hydraulic forces are shown in terms of different arrows. 


$$
\begin{aligned}
R_{k+1} & =R_{k}+\tau V_{k}+\frac{\tau^{2}}{2 M} F^{t o t} \\
V_{k+1} & =V_{k}+\frac{\tau}{M} F^{t o t},
\end{aligned}
$$

where $\tau, k, M$ and $V$ denote the time grid, the time step, the mass of the intruder and the velocity fields, respectively. To facilitate better numerical outcomes, multistep technique [22] is also used in significant force regimes.

\section{Numerical Results and Discussion}

In this section, we carry out some numerical results as given in Section 2. Parameters adopted in this paper could be found in [17]. By substituting them into Equations (2)-(6) and assuming the room temperature of $T=300 \mathrm{~K}$, we determine the total forces consisting of both van der Waals and electrostatics forces for a water molecule and a sodium ion with the total local surface charge $Q=0$ e and 0.02e. 100 different water molecule's orientations are considered and the total forces for water molecule and the sodium ion permeating into the functionalized graphene are shown in Figure 2.

Here we ignore the case of chloride ion as the ionic size of the ion is larger than that of the pore size so that chloride ions are always repelled from the graphene. From Figure 3, for two proposed $Q$, both the water and sodium ion experience repulsive forces but occur in different magnitudes when they approach the graphene. Without any external forces, both the water and the sodium ion could not penetrate through the graphene. While the external forces are applied into the system, the salt water will be pushed towards the sheet in order to overcome the repulsive forces induced by the graphene. Since the repulsive forces of the sodium ion are stronger than that of the water in the case of the neutral graphene, there must exist a range of the external forces, so that for a given container size $R_{0}{ }^{3}$, the water molecule will get through the graphene while the sodium ion will be rejected from the graphene. Once the water (or the sodium ion) reach the pore center, the repulsive forces generated by the permeate side of the graphene will automatically suck them out of the sheet so that the acceptance criteria for the desalination could be written as

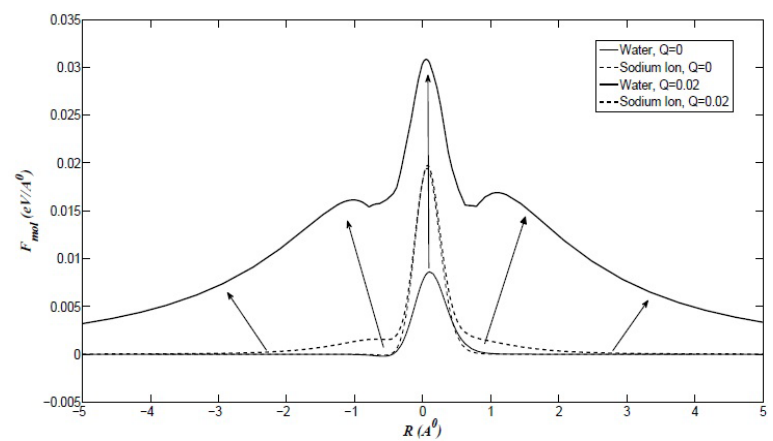

Figure 2. Total forces of the water molecule and sodium ion entering the functionalized graphene where the positive forces indicate the repulsive forces generated by the graphene for $Q=0$ e (neutral) and $0.02 \mathrm{e}$. The arrow indicates the growth of repulsive forces for water molecule when positive charge of 0.02e is doped on the boundary of the pore vicinity.
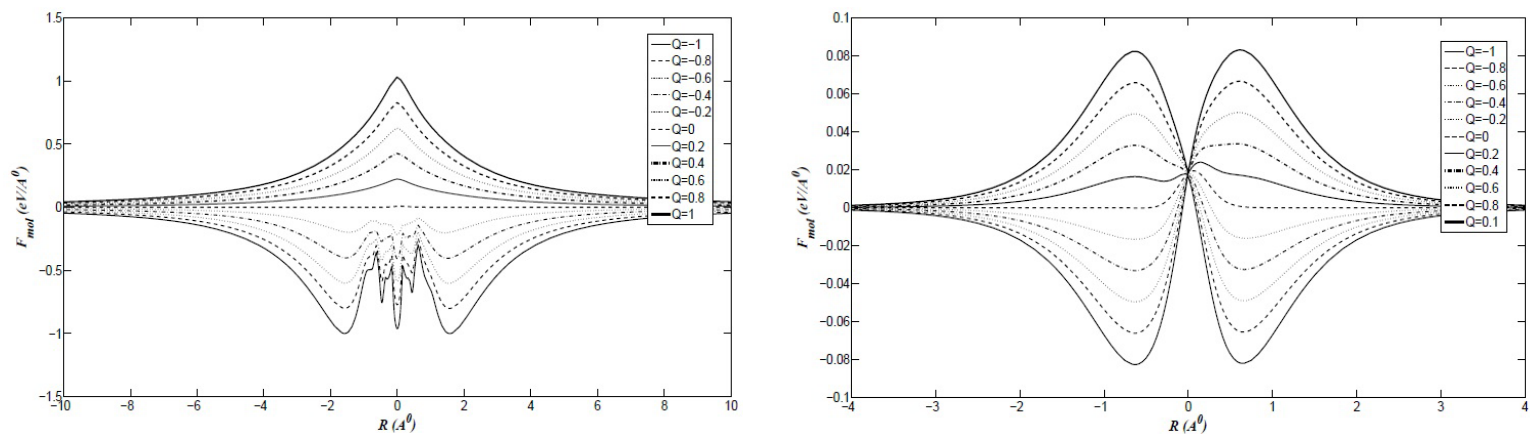

Figure 3. Total forces of the water and sodium ion penetrating into the functionalized graphene for different $Q$. 


$$
\int_{0}^{R_{0}} F_{I} d R>\int_{0}^{R_{0}} F_{\text {ext }} d R>\int_{0}^{R_{0}} F_{W} d R,
$$

where $R_{0}, F_{I}$ and $F_{W}$ denote the initial position of the intruder, the ionic forces of the sodium ion and the molecular forces of the water molecule, respectively. For neutral graphene, we have the following findings: There exists an energy gap between the water molecule and the sodium ion so that given the external forces that satisfy Equation (8), only water will pass through the graphene resulting in desalination. The larger the strength of the hydraulic and the size of the container, the lower the feasibility for the desalination, especially thermal fluctuation is taken into account. However, when tiny charges for example $Q=0.02 \mathrm{e}$ is present on the pore surface, the validity of Equation (8) is broken due to the fact that the water encounters stronger repulsive forces than that of the sodium ion (See Figure 2). In this case, the desalination fails. To demonstrate such effect, we plot the total forces of the water and the sodium ion, where $Q$ ranges from -1e to 1e in Figure 3.

The irregular force fields of water result from the averaging process in Equation (5). For positively charged graphene, water molecule tends to experience larger repulsive forces than that of the sodium ion due to the interaction between the two outer hydrogens and the functionalized graphene. Due to the symmetry of the sodium ion, all electrostatics forces are eliminated at the pore center for all $Q$ resulting in a meeting point of the total forces given in Figure 3. We observe that the functionalized graphene always accepts counter ion.

Now, the force fields of both the water molecule and sodium ion are inserted into the MD simulation frameworks, i.e. Equation (7) to investigate the charge effect on the desalination. Initially, we assume constant external forces $F_{e x t}=4 \mathrm{e}^{-4} \mathrm{eV} / \AA$ and container size of $R_{0}{ }^{3}=10 \AA^{3}$ for which two different total charge on the pore vicinity, i.e. $Q=0$ e and 0.02e are considered. From Equation (8), when $F_{\text {ext }}$ is between $3.547 \mathrm{e}^{-4}$ and $6.047 \mathrm{e}^{-4} \mathrm{eV} / \AA$, only water could penetrate through the neutral graphene, which is confirmed by Figure 4.

However when the graphene sheet is doped by a tiny charge $Q=0.02 \mathrm{e}$ and under the same external force of $4 \mathrm{e}^{-4} \mathrm{eV} / \AA$, both water and sodium ion are blocked by the positively charged graphene. The path of the sodium ion with and without functional group collides almost with each other's which could be partially explained by the comparable repulsive force of the sodium ion as shown in Figure 2. In other words, the positively charged graphene induces more repulsive forces on water molecule than that on sodium ion. However, when $F_{\text {ext }}$ lies between $1.7 \mathrm{e}^{-3}$ and $12.5 \mathrm{e}^{-3} \mathrm{eV} / \AA$, a peculiar phenomenon occurs, where only sodium ion could pass through the positively charged graphene leaving both water and chloride ions on the feed side, which is again demonstrated in Figure 4. To summarize, negatively charged graphene produces acidic solution on the permeate side; small doped positive charges on the graphene severally hamper the efficiency of the desalination; and for certain external forces, mere sodium ions could pass through the positively charged graphene.

\section{Conclusion}

In this paper, we adopt both the continuous approximation and MD simulations to investigate the desalination using the functionalized graphene. Small positive surface charges are shown to severely affect its desalination functionality. More intriguingly, while the negatively charged graphene produces acidic solution on the permeate side, for certain ranges of external forces, only sodium ions are sucked into the positively charged graphene. The present methodology has the merit of rapid computational speed and generates inductive results, where the total forces are determined using surface and line integrals.

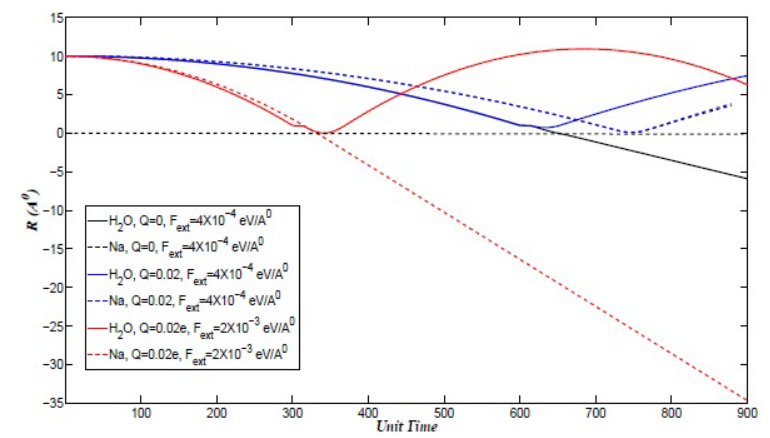

Figure 4. MD simulations for the permeation of water and sodium ion into the graphene with different $F_{\text {ext }}$ and $Q=0 \mathrm{e}$ and $0.02 \mathrm{e}$. 


\section{Acknowledgements}

We gratefully acknowledge the constructive discussion with Dr. Yung-Tsang Chen, and the financial support from small research grant (UNNC), Ningbo Natural Science Foundation (2014A610025), (2014A610172) and (2015A610281), Zhejiang Natural Science Foundation (Q15E090001) and Qianjiang Talent Scheme (QJD1402009).

\section{References}

[1] Severin, K. (2009) Boronic Acids as Building Blocks for Molecular Nanostructures and Polymeric Materials. Dalton Trans., 27, 5254-5264. http://dx.doi.org/10.1039/b902849h

[2] Hilder, T., Gordon, D. and Chung, S. (2009) Salt Rejection and Water Transport through Boron Nitride Nanotubes. Small, 5, 2183-2190. http://dx.doi.org/10.1002/smll.200900349

[3] Hummer, G., Rasaiah, J.C. and Noworyta, J.P. (2001) Water Conduction through the Hydrophobic Channel of a Carbon Nanotube. Nature, 414, 188-190. http://dx.doi.org/10.1038/35102535

[4] Corry, B. (2008) Designing Carbon Nanotube Membranes for Efficient Water Desalination. J. Phys. Chem. B, 112, 1427-1434. http://dx.doi.org/10.1021/jp709845u

[5] Song, C. and Corry, B. (2009) Intrinsic Ion Selectivity of Narrow Hydrophobic Pores. J. Phys. Chem. B, 113, 76427649. http://dx.doi.org/10.1021/jp810102u

[6] Berezhkovskii, A. and Hummer, G. (2002) Single-File Transport of Water Molecules through a Carbon Nanotube. Phys. Rev. Lett., 89, Article ID: 064503. http://dx.doi.org/10.1103/physrevlett.89.064503

[7] Zuo, G., Shen, R., Ma, S. and Guo, W. (2010) Transport Properties of Single-File Water Molecules inside a Carbon Nanotube Biomimicking Water Channel. ACS Nano, 4, 205-210. http://dx.doi.org/10.1021/nn901334w

[8] Kalra, A., Garde, S. and Hummer, G. (2003) Osmotic Water Transport through Carbon Nanotube Membranes. PNAS, 100, 10175-10180. http://dx.doi.org/10.1073/pnas.1633354100

[9] Sansom, M.S.P., Shrivastava, I.H., Ranatunga, K.M. and Smith, G.R. (2000) Simulations of Ion Channels Watching Ions and Water Move. Trends. Biochem. Sci., 25, 368-374. http://dx.doi.org/10.1016/S0968-0004(00)01613-3

[10] Theresa, M., Pendergast, M. and Hoek, E.M.V. (2011) A Review of Water Treatment Membrane Nanotechnologies. Energy Environ. Sci., 4, 1946-1971. http://dx.doi.org/10.1039/c0ee00541j

[11] Chan, Y. (2015) Mathematical Modeling and Simulations on Massive Hydrogen Yield Using Functionalized Nanomaterials. J. Math. Chem., 53, 1280-1293. http://dx.doi.org/10.1007/s10910-015-0488-4

[12] Tanugi, D.C. and Grossman, J.C. (2012) Water Desalination across Nanoporous Graphene. Nano Lett., 12, $3602-3608$. http://dx.doi.org/10.1021/nl3012853

[13] Choi, J.Y. (2013) A Stamp for All Substrates. Nature, 8, 311-312.

[14] Cox, B.J., Thamwattana, N. and Hill, J.M. (2007) Mechanics of Atoms and Fullerenes in Single-Walled Carbon Nanotubes. I. Acceptance and Suction Energies. Proc. R. Soc. London, Ser. A, 463, 461. http://dx.doi.org/10.1098/rspa.2006.1771

[15] Cox, B.J., Thamwattana, N. and Hill, J.M. (2007) Mechanics of Atoms and Fullerenes in Single-Walled Carbon Nanotubes. II. Oscillatory Behavior. Proc. R. Soc. London, Ser. A, 463, 477. http://dx.doi.org/10.1098/rspa.2006.1772

[16] Chan, Y. and Hill, J.M. (2011) A Mechanical Model for Single-File Transport of Water through Carbon Nanotube Membranes. J. Membrane Sci., 372, 57-65. http://dx.doi.org/10.1016/j.memsci.2011.01.040

[17] Chan, Y. and Hill, J.M. (2012) Modeling on Ion Rejection Using Membranes Comprising Ultra-Small Radii Carbon Nanotubes. Eur. Phys. J. B., 85, 56. http://dx.doi.org/10.1140/epjb/e2012-21029-0

[18] Chan, Y. and Hill, J.M. (2013) Ion Selectivity Using Membranes Comprising Functionalized Carbon Nanotubes. J. Math. Chem., 51, 1258-1273. http://dx.doi.org/10.1007/s10910-013-0142-y

[19] Chan, Y. (2015) Mathematical Modeling on Seawater Desalination Using Nanomaterials. Materials Today: Proceedings, 2, 113-117. http://dx.doi.org/10.1016/j.matpr.2015.04.018

[20] Chan, Y. (2015) Mathematical Modeling and Simulations on Massive Hydrogen Yield Using Functionalized Nanomaterials. J. Math. Chem., 53, 1280-1293. http://dx.doi.org/10.1007/s10910-015-0488-4

[21] Sprik, M., Klein, M.L. and Watanable, K. (1990) Solvent Polarization and Hydration of the Chlorine Anion. J. Phys. Chem., 94, 6483-6488. http://dx.doi.org/10.1021/j100379a060

[22] Pang, T. (2006) An Introduction to Computational Physics. 2th Edition, Cambridge University Press, Cambridge. http://dx.doi.org/10.1017/CBO9780511800870 